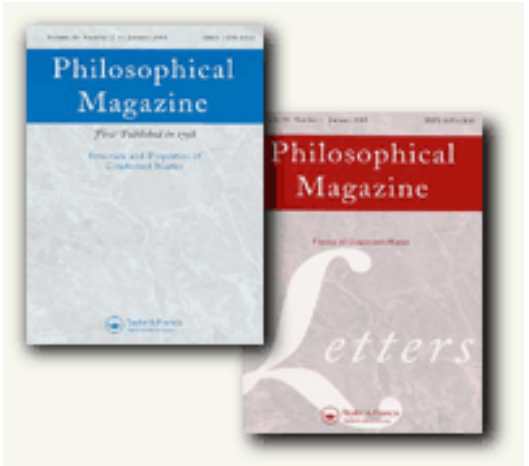

\title{
Czochralski growth and X-ray topographic characterization of decagonal AICoNi quasicrystals
}

\begin{tabular}{|r|l|}
\hline Journal: & Philosophical Magazine \& Philosophical Magazine Letters \\
\hline Manuscript ID: & TPHM-05-May-0197.R1 \\
\hline Journal Selection: & Philosophical Magazine \\
\hline Author: & 05-Jul-2005 \\
\hline & $\begin{array}{l}\text { Complete List of Authors: } \\
\text { Cestkörperforschung } \\
\text { Crysterernst, Götz; Ludwig-Maximilians-Universität München, } \\
\text { Haertwig, Juergen; ESRF } \\
\text { Schenk, Thomas; ESRF } \\
\text { Gille, Peter; Ludwig-Maximilians-Universität München, } \\
\text { Crystallography Section }\end{array}$ \\
\hline Keywords (user supplied): & AlCoNi, Czochralski growth \\
\hline & \\
\hline Keywords: & \\
\hline
\end{tabular}

\section{S) ScholarONE \\ Manuscript Central}


Czochralski growth and X-ray topographic characterization of decagonal AICoNi quasicrystals,

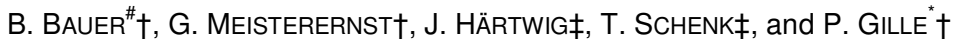

†Crystallography Section, Ludwig-Maximilians-Universität München, Germany ‡E.S.R.F. Grenoble, France

\begin{abstract}
Large single crystals of the decagonal AICoNi phase were reproducibly grown by the Czochralski method from Al-rich melts. By using native seeds, it was possible to grow crystals with well-defined orientations, e.g. along the 10 -fold periodic axis or parallel to one of the 2-fold axes. Quasicrystals, that had been pulled in periodic or quasiperiodic directions, were characterized by X-ray transmission topography at the synchrotron source (ESRF) and at laboratory sources using the Lang technique. Because of the metallurgical homogeneity of the grown quasicrystals, lattice parameter differences did not contribute to the topographic contrast, instead, orientation contrast dominated. A column-like substructure along the periodic direction could be revealed. From this it is concluded, that crystal growth along a two-fold direction might be favourable with respect to minimizing defect spreading.
\end{abstract}

Keywords: Quasicrystal; AlCoNi; Czochralski growth; X-ray topography

\title{
§1. INTRODUCTION
}

Decagonal quasicrystals in the ternary Al-Co-Ni system are known to be stable phases [1] that combine periodic and aperiodic types of order in one single crystal. This is the main reason for samples with 10-fold rotational symmetry being particularly interesting for various investigations in solid state physics. Because of the thermodynamic stability of the decagonal AICoNi phase, conventional crystal growth techniques can be used to grow large single crystals. From the phase diagram studies by Gödecke [2] the primary crystallization field, the liquidus surface, and the offstoichiometric equilibrium between the decagonal phase (D) and an Al-rich melt are well known. All techniques utilized so far to grow D-phase AICoNi single crystals make use of the same thermodynamic equilibrium independent of whether they are called Floating zone technique [3], Bridgman method, Flux growth [4], or Czochralski technique [5]. For several reasons the last one may be regarded as the most powerful method. A fairly homogeneous component distribution [6], an easy seeding technique for well-defined growth orientations [7] as well as good observation access to the growth process are the main advantages. Especially the growth of rather large single crystals, that are needed for neutron diffraction experiments and some surface investigations, can reproducibly be done by the Czochralski method. It is the aim of this paper to present recent results on the preparation of

\footnotetext{
\# current address: Max-Planck-Institut für Festkörperforschung, Stuttgart, Germany

Author for correspondence. Email: gille@Imu.de.
} 
large AICoNi quasicrystals with special emphasis on the influence of the pulling direction on the structural perfection to be reached.

\section{§2. EXPERIMENTAL DETAILS}

For this study, all crystals were grown from initial melts of composition $\mathrm{Al}_{77} \mathrm{CO}_{6} \mathrm{Ni}_{17}$ and typical charges of $20-30 \mathrm{~g}$ that had been ex-situ synthesized by using a RF heating facility. Synthesis and crystal growth were done in alumina crucibles. Wedge-shaped $\mathrm{Al}_{72} \mathrm{Co}_{9} \mathrm{Ni}_{19}$ seed crystals in the desired orientation were cut from former single crystals using a wire saw. The Czochralski growth apparatus has been modified during the series of experiments. While at the beginning the growth chamber had been constructed from usual high-vacuum components, we used later on a fully metal-sealed UHV chamber to keep the argon atmosphere (about $900 \mathrm{mbar}$ ) free of oxygen traces during the two-week experiments. Although knowing the liquidus temperature of the initial melt from the literature data and additional DTA measurements, the exact seeding temperature was taken from direct observation. After homogenisation, the initial melt was slowly cooled down below the calculated liquidus temperature until the crystallization of first decagonal needles occurred at the surface of the melt. Then, these needles were resolved by slowly increasing the melt temperature with permanent visual observation. Knowing the temperature at which the last needles have been vanished, a short superheating of the melt is necessary before the single-crystalline seed can contact the melt surface at the formerly found temperature. Seeding always results in wetting of the seed. That is, why a remelting of a small part of the seed is necessary to start pulling from ideal seeding conditions. Pulling rates as low as $0.15 \mathrm{~mm} \mathrm{~h}^{-1}$ were used in this study and counter rotation of the crucible and the growing crystal was applied to enhance material transport in the melt which is crucial in incongruent crystal growth. Diameter control was done by carefully modifying the temperature while keeping the pulling rate unchanged. Instead of a fixed melting temperature in the conventional Czochralski growth theory, the changing liquidus temperature has to be taken into account. That is the reason, why a slowly decreasing temperature has to be ensured during the whole growth process in order to compensate for the shift in the melt composition. During a two-week growth run the working temperature has to be lowered by about $60 \mathrm{~K}$ using a progressively increasing rate that depends on the crystal diameter and the residual amount of the melt. It is this cooling rate which allows diameter control. At the end of each experiment, crystal growth was completed by using a very high pulling rate and thereby suddenly separating the crystal from the rest of the melt. Cooling to room temperature was done by rates of 250 to $350 \mathrm{~K} \mathrm{~h}^{-1}$.

After cutting the crystals into slices of defined orientation, which has been adjusted by X-ray diffraction using the Laue method, surface preparation was done by mechanical polishing with diamond paste of decreasing grain size up to $0.25 \mu \mathrm{m}$ followed by a final mechanochemical polishing step using a colloidal silica suspension (Syton OP-S, Struers). For transmission X-ray topography a final thickness of the samples of about $400 \mu \mathrm{m}$ was obtained after polishing both sides.

Compositional profiles were measured by electron-probe microanalysis (EPMA) using a CAMECA SX 50 instrument ( $15 \mathrm{kV}, 10 \mathrm{nA})$ and Al- $\mathrm{K}_{\alpha}, \mathrm{Co}_{0} \mathrm{~K}_{\alpha}$, and Ni- $\mathrm{K}_{\alpha}$ lines. Pure elemental standards were taken for calibration.

\section{Deleted: and chemical}

Deleted: and Syton OP-S (Struers) as the final step. 
X-ray transmission topography was done at the ESRF synchrotron source $(\lambda=0.0516 \mathrm{~nm})$ with a Si 111 monochromator and at a revolving anode (Mo- $\mathrm{K}_{\alpha}$ radiation, $\left.\lambda=0.071 \mathrm{~nm}\right)$ using a Lang technique laboratory equipment. With the Lang technique, the spot size was restricted to $8 \mathrm{~mm}$ (height) $\times 0.3 \mathrm{~mm}$ (width) and a maximum transfer length of $50 \mathrm{~mm}$ was allowed. Due to the $145 \mathrm{~m}$

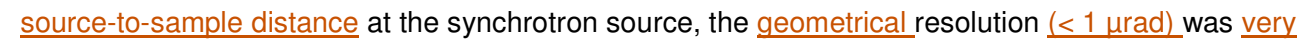
high, but limited to a few $\mu \mathrm{m}$ by the detecting film, while resolutions obtained at the laboratory source were less than 0.5 mrad in-plane and less than 20 mrad in vertical direction. Thus, high resolution images were recorded at the synchrotron source, while routine measurements were done using the Lang technique at the laboratory source. In the latter case, two diffraction images had to be measured with rotating the sample by $90^{\circ}$ in between. Investigating (10000)- as well as (00001)-oriented slices, use was made of 00002-, 10000-, and 10-100 - reflections. Sample adjustment was done with the help of a detector and off-line image plates, but the final images were recorded on high-resolution photographic films (Kodak Industrex SR and AGFA Strukturix D4).

Deleted: much longer collimator, $(145 \mathrm{~m})$

Deleted: much better $(<1 \mu \mathrm{rad})$ than those

Deleted: being

\section{§3. RESULTS AND DISCUSSION}

With the technique described above crystal growth always resulted in single-grain quasicrystals. Typical examples of grown decagonal AICoNi quasicrystals are shown in figure 1. With the first type of the growth chamber having a low but not negligible leakage rate, there were always some traces of oxides on the melt surface that decorated the crystals surface. This is the origin of making 10-fold patterns visible at the periphery of the crystal shown in figure 1a. The quasicrystal pictured in figure $1 \mathrm{~b}$ was grown in the fully metal-sealed chamber that prevents any oxidation and thus gives the crystals a shiny surface. On each of the crystals small facets are to be seen that reveal their single-crystalline nature. Measuring the inclination of all observed facets [8], that well correspond to net planes with the strongest structure amplitudes [9], allows the construction of the idealized decagonal quasicrystal shape that is shown in figure $1 \mathrm{c}$.

Elemental distribution measured by EPMA at a crystal of average composition $\mathrm{Al}_{72} \mathrm{Co}_{9} \mathrm{Ni}_{19}$ has given a very weak axial segregation (see figure 2) with the Co content decreasing by less than 1 at. \% and the Ni content increasing by nearly the same amount. This is well understood from the slope of the tie-lines in the ternary Al-Co-Ni phase diagram [7]. The radial elemental distribution has been measured to be absolutely homogeneous within the error of EPMA. This may be regarded as an essential advantage compared to the Flux method [4], where growth is assumed to proceed mainly in radial direction, thus giving rise to a remarkable radial segregation. Both, axial as well as radial segregation effects in Czochralski-grown AICoNi quasicrystals, have proved to be too weak to contribute to the contrast in X-ray topographic images.

Generally, all X-ray transmission topographs obtained from either synchrotron or laboratory experiments pictured the whole slices under investigation. This has been found with all samples, being longitudinal or radial sections through the crystals. At the synchrotron source, FWHM of rocking curves taken from several $\mathrm{mm}^{2}$ spots were measured to be typically 2 minutes of arc, and topographs were taken during rocking the sample by a total angle of approximately $\alpha=2$ FWHM. Using the Lang technique no sample rocking at all was applied. Figure 3 shows topographic images of axial slices prepared from quasicrystals grown in 10-fold (figure $3 a$ ) and jin one of the 2-fold directions (figure 3b).

Formatted

Deleted: respectively 
These two figures present overview images of the total slices, which have been recorded using the Lang technique with higher resolution parallel to the diffraction plane. Due to the restricted spot height, these images have been composed from several individual pictures. Higher-resolution images from smaller parts of the slices are inserted to judge the structural perfection of those parts, that are regarded as especially critical during growth because of diameter changes. While the single image of the Lang topograph of the crystal grown along [00001] (figure 3a) pretends band shape substructure perpendicular to the growth axis that results from the restricted resolution perpendicular to the diffraction plane, the high-resolution images indicate a rather homogeneous contrast with a system of fine lines throughout the sample being inclined to the pulling direction by an angle of approximately $75^{\circ}$ to both sides. Because of the different diffraction vectors, individual defect types may be differently imaged due to special extinction effects, but the overall images were kept unchanged with all reflections used. Additionally, there is some column-like substructure in the rare part running parallel to the periodic [00001]-direction. In the second crystal (figure 3b) that was grown along the [10000]direction, i.e. with the 10 -fold rotational axis perpendicular to the pulling direction, a rather different contrast has been found. In the overview as well as in the high-resolution images, a cloud-like contrast can be resolved with no preferred distribution in neither of the two quasiperiodic directions. It should be noticed that the line of vision corresponds to the periodic axis [00001]. But, whether or not the weak elliptic figures may be interpreted with respect to an assumed columnar growth along the 10-fold direction this can only be a matter of speculation. The main difference to the first discussed crystal is that no substructure spreading occurs along the crystal axis. From this finding it might be concluded that growth of decagonal AICoNi quasicrystals should preferentially be done in directions being inclined to the 10-fold axis, e. g. along one of the 2 -fold axes as to prevent defect substructures from extending along the whole crystal.

\section{§4. CONCLUSIONS}

We have shown that the Czochralski method has proved to be capable of growing large single-grain decagonal AICoNi quasicrystals of fairly high metallurgical homogeneity. Large-area characterization of the structural perfection of these crystals was possible using X-ray transmission topography at thin quasicrystalline slices. From the results obtained it is suggested to grow single crystals along a 2-fold direction instead of pulling parallel [00001]. In principle, it would be no problem to scale-up the crystal growth technique to grow even larger single crystals. But up to now, to the best of the authors' knowledge, there has been no particular application of single-crystalline decagonal samples that would make this effort meaningful.

\section{ACKNOWLEDGEMENTS}

The authors would like to thank R. Wunderlich for surface preparation and P. Dreier for EPMA measurements. Financial support by the Deutsche Forschungsgemeinschaft under contract Gi 211/6 is gratefully acknowledged. 
REFERENCES

[1] A.P. Tsai, A. Inoue, T. Masumoto, Mater. Trans JIM 30463 (1989).

[2] T. Gödecke, Z. Metallkd. 88557 (1997).

[3] T.J. Sato, T. Hirano, A.P. Tsai, J. Crystal Growth 191545 (1998).

[4] I.R. Fisher, M.J. Kramer, Z. Islam, A.R. Ross, A. Kracher, T. Wiener, M.J. Sailer, A.I. Goldman, P.C. Canfield, Philos. Mag. B 79425 (1999).

[5] P. Gille, P. Dreier, M. Gräber, T. Scholpp, J. Crystal Growth 20795 (1999).

[6] M. Gräber, R.-U. Barz, P. Dreier, P. Gille, Mater. Sci. Eng. 294-296 143 (2000).

[7] P. Gille, R.-U. Barz, L.M. Zhang, in Quasicrystals. Structure and Physical Properties, edited by H.-R. Trebin (WILEY-VCH, Weinheim, 2003).

[8] P. Gille, G. Meisterernst, N. Faber, J. Crystal Growth 275224 (2005).

[9] W. Steurer and A. Cervellino, Acta Crystallogr. A 57333 (2001). 
CAPTION OF FIGURES

Figure 1: Typical decagonal $\mathrm{Al}_{72} \mathrm{Co}_{9} \mathrm{Ni}_{19}$ quasicrystals $(\mathrm{a}, \mathrm{b})$ grown by the Czochralski method along the 10-fold rotational axis and a schematic figure (c) of an idealized decagonal quasicrystal of point group 10/mmm showing all crystallographic forms that have been observed at real AICoNi quasicrystals as facets on as-grown surfaces.

Figure 2: $\quad$ Elemental distribution along the central trace of the decagonal $\mathrm{Al}_{72} \mathrm{Co}_{9} \mathrm{Ni}_{19}$ quasicrystal pictured in figure 1a. EPMA measurement on the same (10000)-oriented slice used for $\mathrm{X}$-ray transmission topography reveals only a marginal shift in $\mathrm{Co}$ and $\mathrm{Ni}$ concentrations that cannot cause differences in the image contrast.

Figure 3: $\quad \mathrm{X}$-ray transmission topographs of longitudinal sections of Czochralski-grown $\mathrm{Al}_{72} \mathrm{Co}_{9} \mathrm{Ni}_{19}$ quasicrystals (g: diffraction vector).

a) (10000)-oriented slice prepared from a crystal that has been grown parallel to the 10 -fold rotational axis. Lang technique image (00002-reflection) and higher-resolution images from smaller parts of the slice (inserts) obtained from synchrotron topography (10100 - reflection),

b) (00001)-oriented section through a quasicrystal grown along [10000] which is one of the two-fold symmetry directions. Lang technique image (10000-reflection) and higher-resolution images from smaller parts of the slice (inserts) obtained from synchrotron topography ( $10 \overline{100}$ - reflection). 

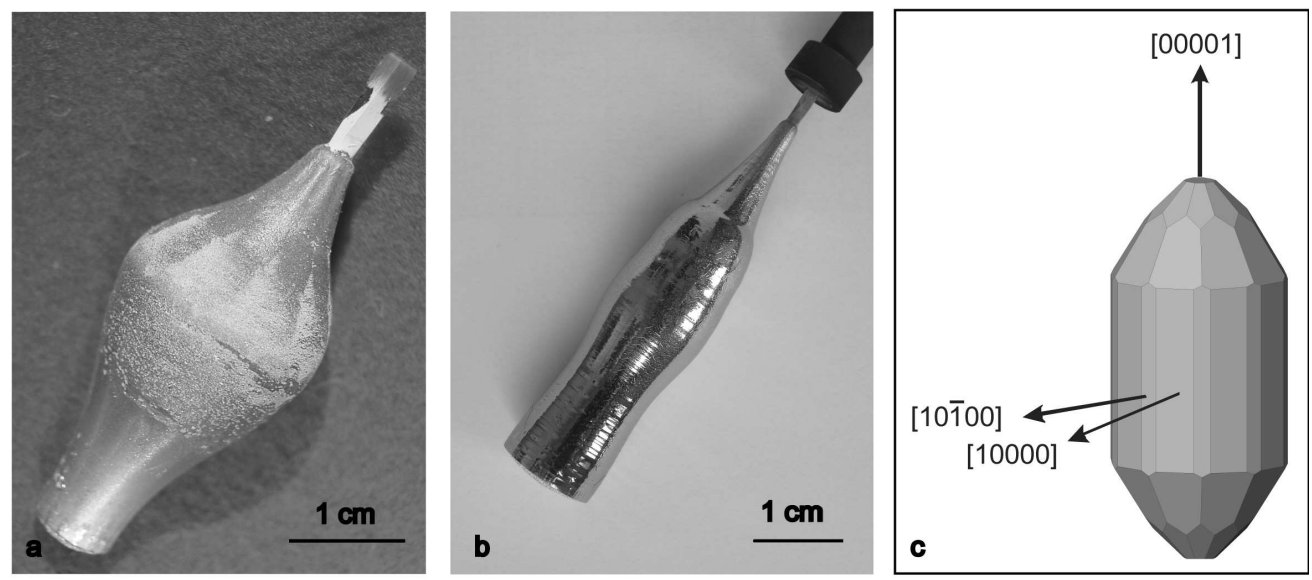

Typical decagonal $\mathrm{Al}_{72} \mathrm{Co}_{9} \mathrm{Ni}_{19}$ quasicrystals $(\mathrm{a}, \mathrm{b})$ grown by the Czochralski method along the 10 fold rotational axis and a schematic figure (c) of an idealized decagonal quasicrystal of point group $10 / \mathrm{mmm}$ showing all crystallographic forms that have been observed at real AlCoNi quasicrystals as facets on as-grown surfaces. $199 \times 87 \mathrm{~mm}(300 \times 300 \mathrm{DPI})$ 


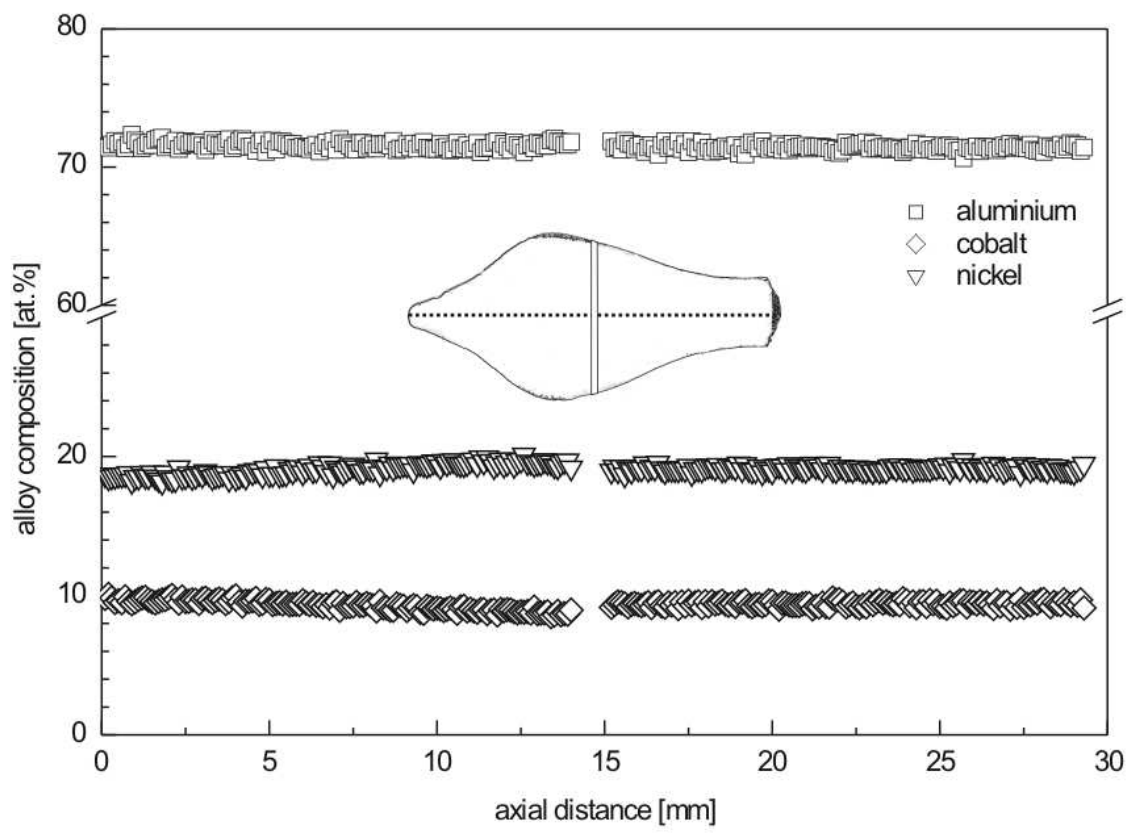

Elemental distribution along the central trace of the decagonal $\mathrm{Al}_{72} \mathrm{Co}_{9} \mathrm{Ni}_{19}$ quasicrystal pictured in figure 1a. EPMA measurement on the same (10000)-oriented slice used for X-ray transmission topography reveals only a marginal shift in $\mathrm{Co}$ and $\mathrm{Ni}$ concentrations that cannot cause differences in the image contrast. 

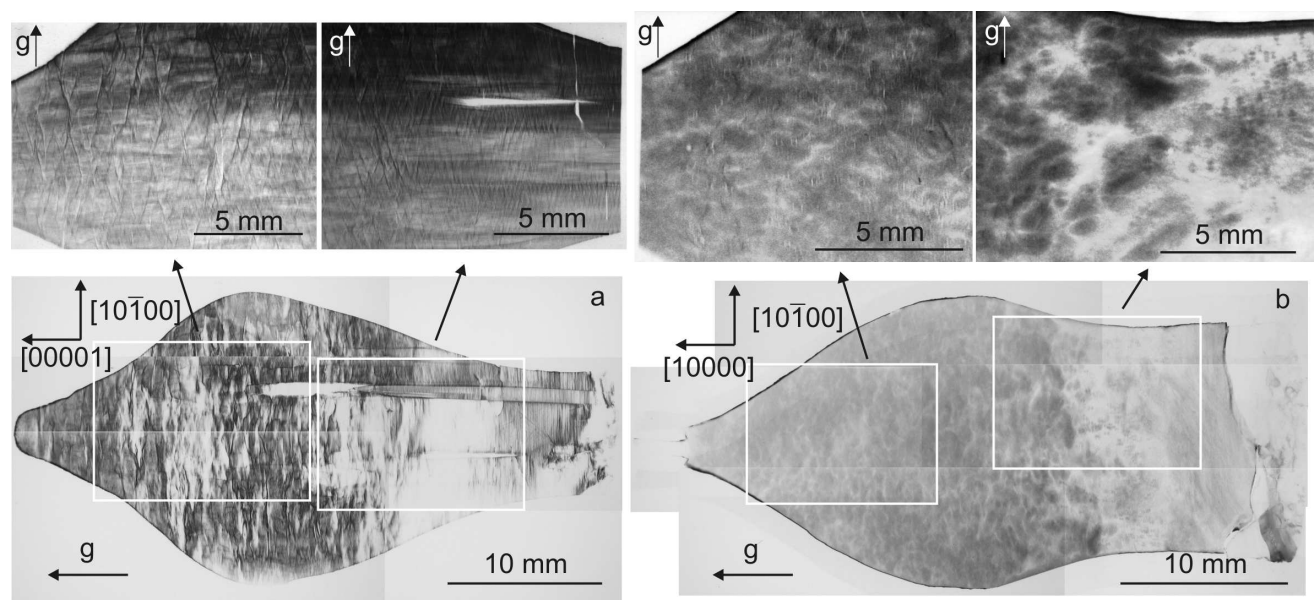

X-ray transmission topographs of longitudinal sections of Czochralski-grown $\mathrm{Al}_{72} \mathrm{Co}_{9} \mathrm{Ni}_{19}$ quasicrystals ( $\mathrm{g}$ : diffraction vector).

a) (10000)-oriented slice prepared from a crystal that has been grown parallel to the 10 -fold rotational axis. Lang technique image (00002-reflection) and higher-resolution images from smaller parts of the slice (inserts) obtained from synchrotron topography (10-100-reflection),

b) (00001)-oriented section through a quasicrystal grown along [10000] which is one of the twofold symmetry directions. Lang technique image (10000-reflection) and higher-resolution images from smaller parts of the slice (inserts) obtained from synchrotron topography (10-100-reflection). 